\title{
A CMOS 15-Bit 125-MS/s Time-Interleaved ADC with Digital Background Calibration
}

\author{
Zwei-Mei Lee, Cheng-Yeh Wang, and Jieh-Tsorng Wu \\ Department of Electronics Engineering, National Chiao-Tung University, Hsin-Chu 300, Taiwan
}

\begin{abstract}
A 15-bit 125-MS/s two-channel time-interleaved pipelined ADC is fabricated in a $0.18 \mu \mathrm{m}$ CMOS technology, and achieves $91.9 \mathrm{~dB}$ SFDR, 69.9 dB SNDR for a $9.99 \mathrm{MHz}$ input. The ADC uses a single sample-and-hold amplifier which employs a precharging circuit technique to mitigate the performance requirements for its opamp. Digital background calibration is applied to maintain the conversion linearity of each $A / D$ channel and also correct both gain and offset mismatches between the two channels. Excluding $\mathrm{I} / \mathrm{O}$ buffers, the chip occupies an area of $4.3 \times 4.3 \mathrm{~mm}^{2}$ and dissipates $909 \mathrm{~mW}$ from a $1.8 \mathrm{~V}$ supply.
\end{abstract}

\section{INTRODUCTION}

High-resolution high-speed Nyquist-rate analog-to-digital converters (ADCs) have been predominantly realized using the pipeline architecture. High-gain opamps with linear feedback are often used to ensure the linearity of sample-and-hold amplifiers and pipeline stages. To achieve more than 14-bit resolution, the opamps are required to have a voltage gain of more than $90 \mathrm{~dB}$, which results in reduced speed. Thus, there are few published ADCs that can achieve both more than 14-bit resolution and more than 100-MS/s sample rate. The BiCMOS ADC of [1] achieves $75 \mathrm{~dB}$ SNR and $100 \mathrm{~dB}$ SFDR at $125 \mathrm{MS} / \mathrm{s}$ sample rate, but also consumes $1.95 \mathrm{~W}$ of power. The BiCMOS ADC of [2] achieves $70.2 \mathrm{~dB}$ SNDR and $79.9 \mathrm{~dB}$ SFDR at $100 \mathrm{MS} / \mathrm{s}$ sample rate by using timeinterleaved architecture with foreground digital calibration. It consumes $1.4 \mathrm{~W}$ of power. The CMOS ADC of [3] employs digital calibration to mitigate the requirements for the opamps. But it achieves only $70 \mathrm{~dB}$ total harmonic distortion (THD) at $100-\mathrm{MS} / \mathrm{s}$ sample rate.

The ADC reported in this paper comprises two timeinterleaving pipelined A/D channels to achieve an equivalent sample rate of $125 \mathrm{MS} / \mathrm{s}$. A single sample-and-hold amplifier (SHA) is used to avoid the sampling phase error between the two channels. The SHA uses a precharging circuit configuration to mitigate its requirements for the opamp which has to operate at maximum clock rate of $125 \mathrm{MHz}$. Digital calibration is employed to maintain the conversion linearity of each A/D channel and also correct both gain and offset mismatches between the two channels. The calibration is proceeded continuously without interrupting the normal A/D operations. The ADC chip was fabricated in a $0.18 \mu \mathrm{m} 1 \mathrm{P} 6 \mathrm{M}$ CMOS technology with MIM capacitors, and operates under a single $1.8 \mathrm{~V}$ supply.

\section{ADC ARCHITECTURE}

Fig. 1 shows the block diagram of the time-interleaved ADC presented in this paper. It consists of a single SHA and two

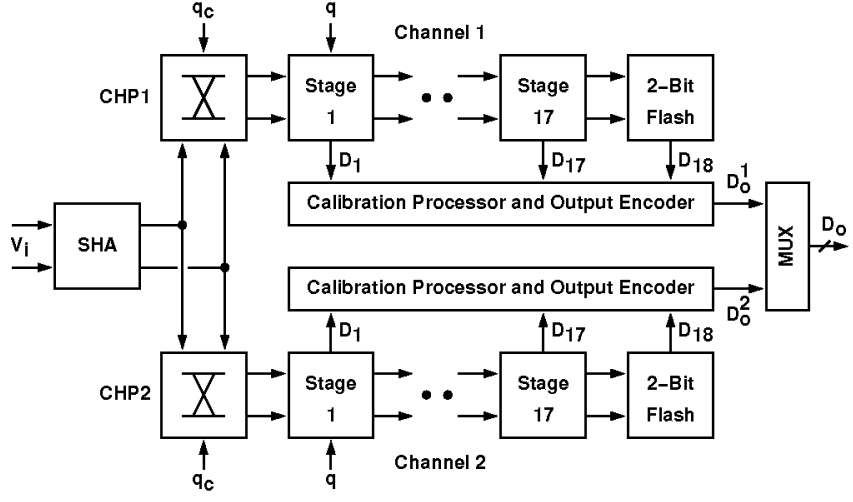

Fig. 1. ADC block diagram.

identical analog-to-digital conversion (A/D) channels. The use of a single SHA avoids any sampling skew error between the two channels. The offset and gain mismatch between the two A/D channels are corrected in the backend digital domain. The final digital output is obtained by multiplexing the outputs from the two A/D channels.

The two random choppers, CHP1 and CHP2, are placed in front of the two A/D channels respectively. The choppers can multiplex the SHA's outputs to perform time-interleaving operation. They also randomize the polarity of the sampled input seen by the two A/D channels, so that the overall offsets of the A/D channels can be calibrated [4]. The choppers are controlled by a binary-valued random sequence, $q_{c}$.

Each A/D channel consists of 17 radix-2 1.5-bit switchedcapacitor (SC) pipeline stages and a final 2-bit flash stage. The pipeline employs a correlation-based digital background calibration scheme to achieve high linearity [5]. A $q$ random sequence is injected into the pipeline to facilitate the calibration.

\section{SAMPLE-AND-HOLD AMPLIFIER (SHA)}

Fig. 2 shows the schematic of a precharged SHA that has been proposed for time-interleaved A/D applications [6]. During the $\phi_{3}$ sample mode $\left(\phi_{3}=1\right.$ and $\left.\phi_{1}=1\right)$, the input not only drives the $C_{i 1}$ and $C_{i 2}$ sampling capacitors but also precharges the SHA's output nodes including the input capacitive loadings of the $\mathrm{A} / \mathrm{D}$ channel $1, C_{L 1}$ and $C_{L 2}$. During the $\phi_{4}$ sample mode $\left(\phi_{4}=1\right.$ and $\left.\phi_{1}=1\right)$, the input capacitive loadings of the $\mathrm{A} / \mathrm{D}$ channel $2, C_{L 3}$ and $C_{L 4}$, are precharged instead. During both sample mode period, the 


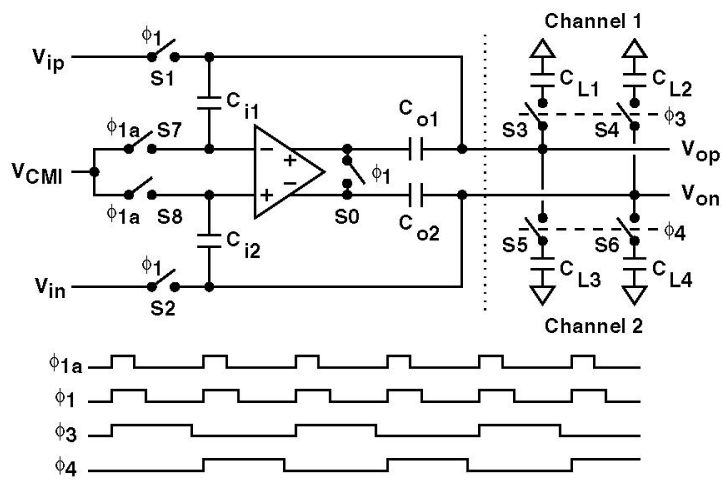

Fig. 2. Precharged sample-and-hold amplifier.
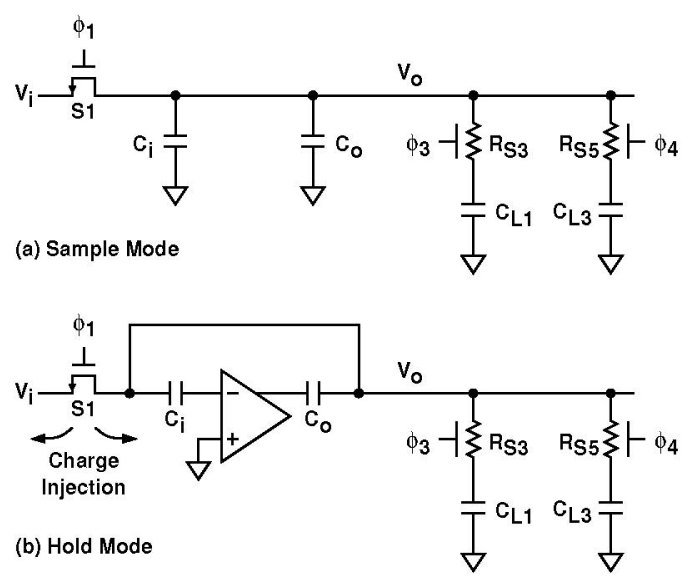

Fig. 3. Simplified SHA operation: (a) sample mode; (b) hold mode.

opamp's outputs are equalized by the $\mathrm{S} 0$ switch, thus, the two output coupling capacitors, $C_{o 1}$ and $C_{o 2}$, are added. When switching to the hold mode $\left(\phi_{1}=0\right)$, the opamp's outputs can settle to their final values in a shorter time period without slewing due to the precharging. The combination of output capacitor coupling and precharging also reduces the opamp's $\mathrm{dc}$ gain and output voltage swing requirements so that higher speed can be achieved.

However, the linearity of the precharged SHA shown in Fig. 2 can be degraded by the mismatches between the S3-S4 output network and the S5-S6 output network. Fig. 3 shows the SHA's single-ended circuit configuration during the sample mode and the hold mode. The S3 and S5 switches are modeled as two voltage-controlled resistors, $R_{S 3}$ and $R_{S 5}$. During the $\phi_{3}$ sample phase, the $R_{S 3}-C_{L 1}$ network is connected to the $V_{o}$ node. During the $\phi_{4}$ sample phase, the $R_{S 5}-C_{L 3}$ network is connected to the $V_{o}$ node. If there are mismatches between the $R_{S 3}-C_{L 1}$ and $R_{S 5}-C_{L 3}$ networks, the $V_{i}$-to- $V_{o}$ frequency response in the sample node is varied when the SHA alternates between the $\phi_{3}$ and $\phi_{4}$ phases. Furthermore, when the SHA is being switched from the sample mode to the hold mode, charges are injected into the $V_{o}$ node with the $\mathrm{S} 1$ switch being turned off. If there are mismatches between the $R_{S 3}-C_{L 1}$ and $R_{S 5}-C_{L 3}$ networks, the amount of $V_{0}$ change due to the charge

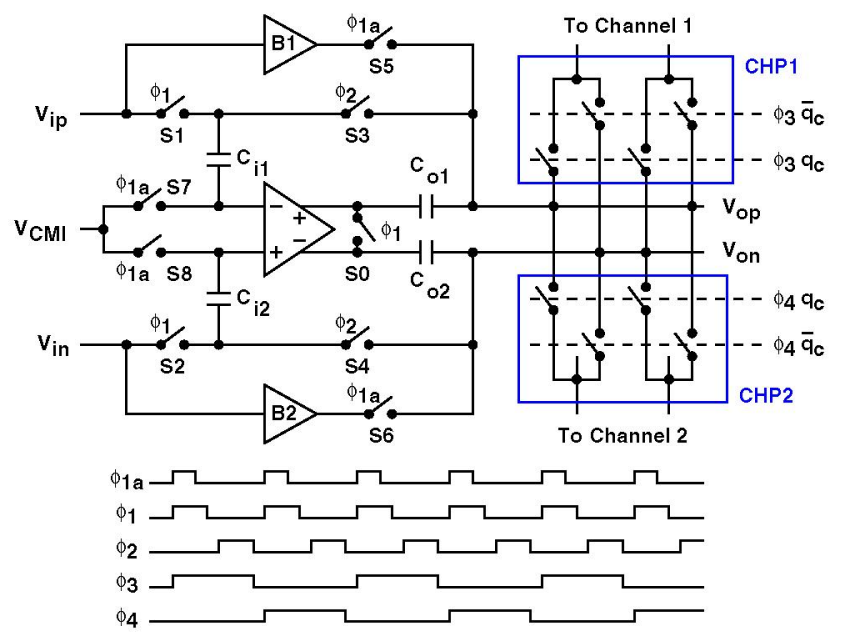

Fig. 4. Modified precharged sample-and-hold amplifier.

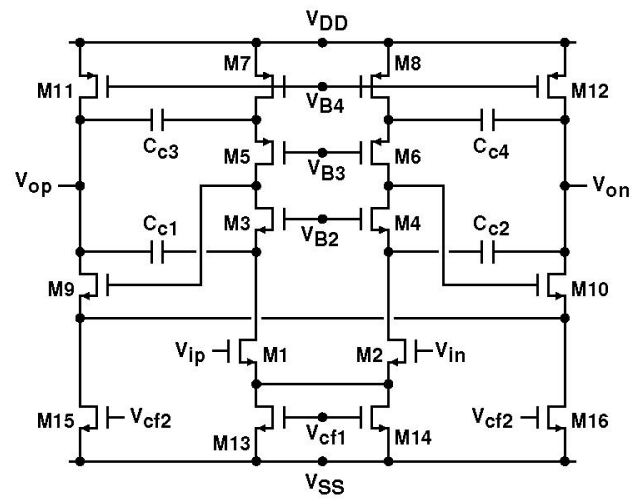

Fig. 5. SHA's operational amplifier.

injection in the $\phi_{3}$ phase is different from that in the $\phi_{4}$ phase.

Fig. 4 shows the circuit schematic of the modified precharged SHA used in this ADC design. Two unity-gain buffers, B1 and B2, are added so that the $V_{i}$-to- $V_{o}$ frequency response can remain the same during either the $\phi_{3}$ sample phase or the $\phi_{4}$ sample phase. The charge injection from the $S 1$ and $\mathrm{S} 2$ switches can no longer affect the final $V_{o}$ output in the hold mode. The charge injections from the additional S5 and S6 switches as well as mismatches between the output multiplexer networks have little effect on the final $V_{o}$ value during the hold mode. Also shown in Fig. 4 are the CHP1 and CHP2 choppers. This SHA samples the input using the $\phi_{1}$ clock with sampling rate at $f_{S}=125 \mathrm{MS} / \mathrm{s}$. Each chopper consists of 4 analog switches controlled by either $\phi_{3}$ or $\phi_{4}$ clocks with frequency at $f_{s} / 2$. Their outputs are inverted when $q_{c}=0$.

Fig. 5 shows the schematic of the opamp used in the SHA. The fully differential two-stage configuration consists of a telescopic first stage followed by a common-source second stage. Two separate switched-capacitor common-mode feedback circuits are used to generate control voltage $V_{c f 1}$ and $V_{c f 2}$ for the first and second stages of the opamp. The 


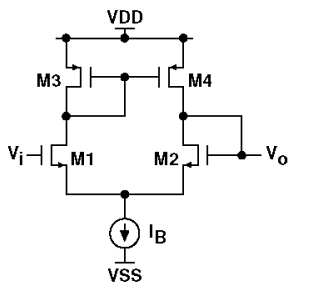

Fig. 6. SHA's unity-gain buffer.

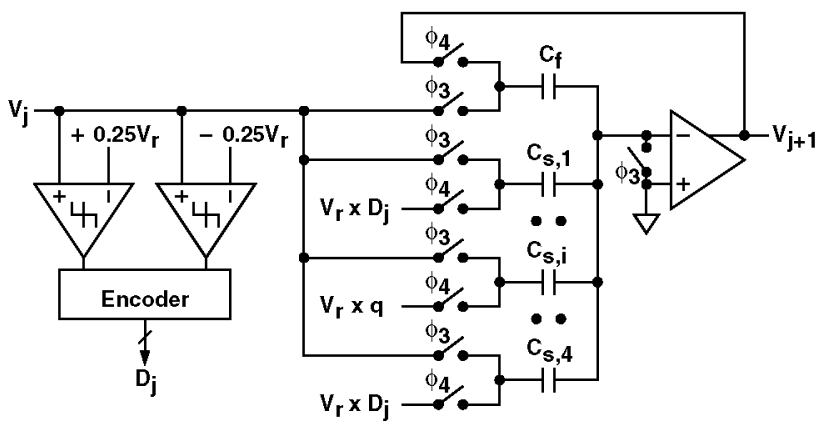

Fig. 7. SC pipelined stage for digital background calibration.

input common-mode voltage is set to $0.95 \mathrm{~V}$, and the output common-mode voltage is set to $1.15 \mathrm{~V}$. The opamp can provide a differential output voltage range of $1.9 \mathrm{~V}$. The overall dc voltage gain of the opamp is more than $90 \mathrm{~dB}$. The opamp dissipates $100 \mathrm{~mW}$ of power and achieves a unity-gain frequency of $1.66 \mathrm{MHz}$ with $C_{i}=4 \mathrm{pF}, C_{o}=6 \mathrm{pF}$, and $C_{L}=4 \mathrm{pF}$.

Fig. 6 shows the schematic of the B1 and B2 unity-gain buffers. It is a simple single-stage amplifier with an open-loop $\mathrm{dc}$ voltage gain of $32 \mathrm{~dB}$. Each buffer dissipates $13 \mathrm{~mW}$ of power while driving a total output capacitive load of $7 \mathrm{pF}$, and has a unity-gain frequency of $2.3 \mathrm{GHz}$ and a slew rate of $1 \mathrm{~V} / \mathrm{nsec}$. The behavior of the buffers can deviate from an ideal one due to the offset and low open-loop voltage gain of the amplifier. This non-ideal buffer behavior is not crucial, but the deviation results in a less accurate precharge operation, and demands an increase in the opamp's voltage gain to maintain the SHA's accuracy.

\section{Calibration AND Digital ENCODING}

The digital background calibration described in [5] is used to ensure the conversion linearity of each A/D channel. To facilitate background calibration, the first six pipeline stages employ the circuit schematic shown in Fig. 7. The capacitor $C_{S}$ is equally split into 4 fragments, $C_{s, 1}$ to $C_{s, 4}$. For an ideal radix-2 pipeline stage, $C_{f}=C_{S}=\sum_{i=1}^{4} C_{S, i}$ During the sample phase $\left(\phi_{3}=1\right)$, the $V_{j}$ stage input is stored in $C_{s}$ and $C_{f}$ capacitors and is also compared with the $\pm 0.25 V_{r}$ references to generate a $D_{j}$ stage digital code, where $D_{j} \in\{-1,0,+1\}$. During the hold phase $\left(\phi_{4}=1\right)$, all the $C_{S}$ capacitors are connected to $D_{j} \times V_{r}$ except the $C_{s, i}$ capacitor which is connected to $q \times V_{r}$. The $q$ random sequence alternates between +1 and 0 or between -1 and 0 . By injecting a random

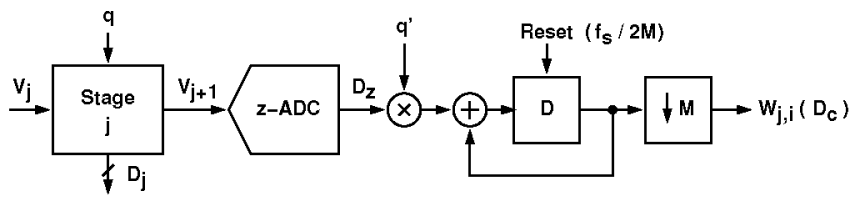

Fig. 8. Background calibration of pipeline stage.

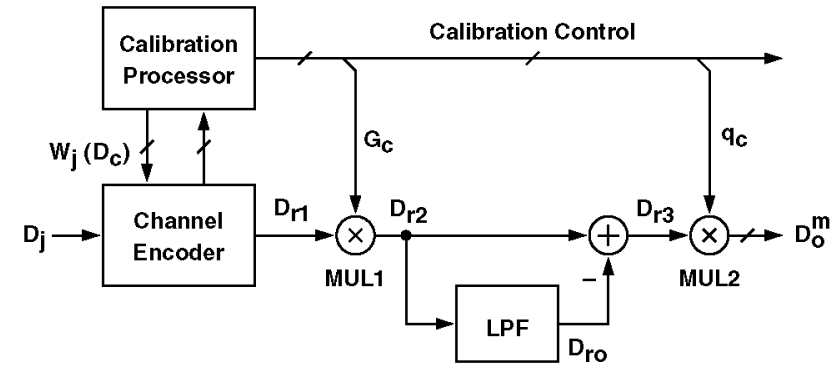

Fig. 9. Digital background calibration scheme.

sequence into the pipeline stage, it is possible to calibrate a pipeline stage without interrupting its normal A/D operation.

Fig. 8 shows the scheme of digital background calibration. The $V_{j+1}$ output of the $j$-th pipeline stage is digitized by a backend $\mathrm{z}$-ADC with a corresponding $D_{z}$ digital output. The Z-ADC comprises of the $(j+1)$-th, $(j+2)$-th, $\ldots$, pipeline stages. The $D_{z}$ signal is correlated with a $q^{\prime}$ random sequence and then integrated on an accumulator. The $q^{\prime}$ signal has the same waveform pattern as the $q$ sequence but alternates between +1 and -1 . The resulting output $W_{j, i}\left(D_{c}\right)$ is taken only after $M$ cycles of integration, where $M$ is the period of the $q$ random sequence. In one $M$ period, the $q^{\prime}$ sequence needs to have equal number of +1 and -1 . The $W_{j, i}\left(D_{c}\right)$ represents the magnitude of the $q$ random sequence seen by the $\mathrm{z}-\mathrm{ADC}$, where $D_{c}$ is either +1 or -1 depending on the polarity of the $q$ sequence. The final calibration data, $W_{j}\left(D_{c}\right)$ where $D_{c} \in\{-1,0,+1\}$, are assembled by letting $W_{j}\left(D_{c}\right)=\sum_{i=1}^{4} W_{j, i}\left(D_{c}\right)$ and $W_{j}(0)=0$.

Assume there are $P$ pipeline stages in each A/D channel, and only the first $K$ stage are subjected to calibration. For $j>K$, we can choose $W_{j}\left(D_{c}\right)=D_{c}$. Calibration is preceded backward and sequentially while the ADC performing normal A/D conversion. First, the $K$-th stage is calibrated using the backend stages, from $(K+1)$ to $P$, as the $\mathrm{z}$-ADC to extract the $W_{K}\left(D_{c}\right)$ data. Then, the $(K-1)$-th stage is calibrated using the backend stages, from $K$ to $P$, as the z-ADC to extracted the $W_{K-1}\left(D_{c}\right)$ data. The procedure continues toward the first stage to complete one calibration cycle.

Fig. 9 shows the block diagram for output encoding and correction in each A/D channel. The channel encoder calculates the raw A/D output, $D_{r 1}$, from pipeline stage's digital outputs, $D_{j}$ for all $j$, by applying

$$
D_{r 1}=\sum_{j=1}^{p} \frac{W_{j}\left(D_{j}\right)}{2^{j}}
$$

The $D_{r 1}$ stream is free of nonlinearities caused by the gain 


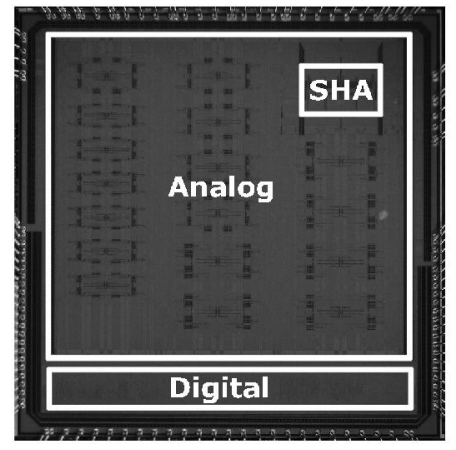

Fig. 10. Die micrograph of the prototyping ADC.

errors of the pipeline stages. To equalize the conversion gains of the two A/D channels, the MUL1 multiplier multiplies the $D_{r 1}$ with a $G_{c}$ gain correction factor, and generates the $D_{r 2}$ stream. The calibration processor shown in Fig. 9 calculates the $G_{c}$ factor from the acquired calibration data by using

$$
G_{c}=\frac{2 \times \sum_{j=1}^{P} \frac{1}{2^{\prime}}}{\sum_{j=1}^{P} \frac{W_{j}(+1)}{2^{j}}-\sum_{j=1}^{P} \frac{W_{j}(-1)}{2^{j}}}
$$

The overall $\mathrm{dc}$ offset of the A/D channel, $D_{r o}$, is extracted from the $D_{r 2}$ stream by a low-pass filter (LPF) [4]. The $D_{r 3}$ stream is the $D_{r 2}$ stream with its de offset removed. Finally, the single channel output, $D_{o}^{m}$ where $m \in\{1,2\}$, is obtained by unscrambling the $D_{r 3}$ stream with the $q_{c}$ sequence.

\section{EXPERIMENTAL RESULTS}

Fig. 10 shows the ADC's chip micrograph. Chip core area is $4.3 \times 4.3 \mathrm{~mm}^{2}$. Digital circuits occupy $11 \%$ of the total area. Operating at $125 \mathrm{MS} / \mathrm{s}$ sampling rate under a single $1.8 \mathrm{~V}$ supply, the analog block consumes a total of $891 \mathrm{~mW}$ of power, while the digital block consumes only $18 \mathrm{~mW}$. In this design, the first six pipeline stages of both A/D channels are implemented with identical capacitors and opamps. The nominal values of the capacitors are $C_{f}=2 \mathrm{pF}$ and $C_{s}=2 \mathrm{pF}$. The opamps are similar to the one shown in Fig. 5, and each consume $47 \mathrm{~mW}$ of power. The opamps and capacitors are reduced by half in the next five stages. Another scaling by half is applied to the remaining stages. This ADC prototype was not optimized for low power consumption.

Fig. 11 shows the measured differential nonlinearity (DNL) and integral nonlinearity (INL) of the ADC operating at $125 \mathrm{MS} / \mathrm{s}$ sample rate with $1.99 \mathrm{MHz}$ sinusoidal input. Digital output codes from a single channel are collected to calculate the DNL and INL. Note that the LSB in Fig. 11 is normalized to 16-bit resolution. The number of registered output codes is approximately $(3 / 4) \times 2^{16}$. Before activating the calibration processor, the native DNL is $+1.16 /-0.61 \mathrm{LSB}$ and the INL is $+29.8 /-29.7$ LSB. After the background calibration is activated. The DNL is reduced to $+0.25 /-0.27 \mathrm{LSB}$ and the INL is reduced to $+5.5 /-5.7 \mathrm{LSB}$.

Fig. 12 shows the ADC's measured signal-to-distortionplus-noise (SNDR) and spurious-free dynamic range (SFDR)
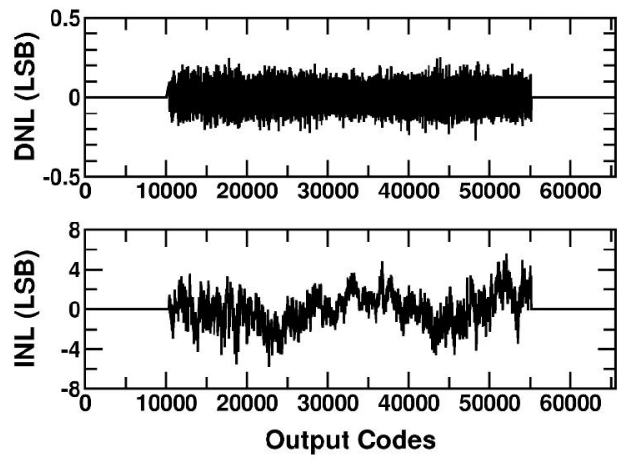

Fig. 11. Measured DNL and INL.

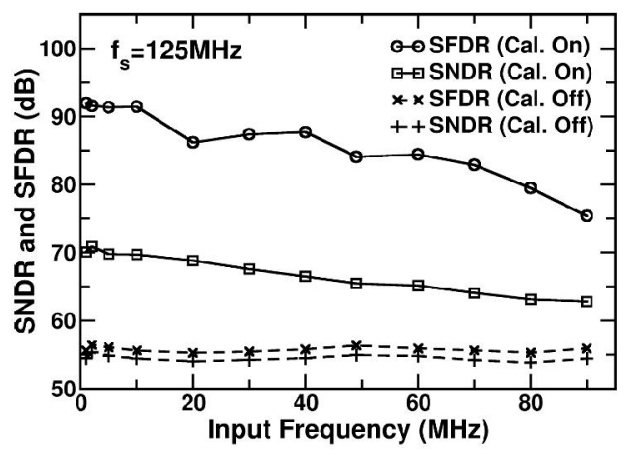

Fig. 12. Measured SNDR and SFDR versus input frequencies.

versus input frequencies at $125 \mathrm{MS} / \mathrm{s}$ sampling rate. The calibration can improve the SNDR by more than $10 \mathrm{~dB}$ and the SFDR about $30 \mathrm{~dB}$ when the input frequencies up to the Nyquist frequency.

\section{ACKNOWLEDGEMENTS}

This work was supported by the National Science Council of Taiwan, R.O.C. (Contract No. NSC-94-2215-E-009-045), and the MediaTek Research Center at National Chiao-Tung University (NCTU). The authors also thank the Si2 Laboratory at NCTU for supporting chip fabrication.

\section{REFERENCES}

[1] A. M. Ali, C. Dillon, R. Sneed, A. Morgan, J. Kornblum, L. Wu, and S. Bardsley, "A 14-bit 125MS/s IF/RF sampling pipelined A/D converter," IEEE Custom Integrated Circuits Conference, pp. 10-4-1$10-4$ - September 2005.

[2] V. Hakkarainen, M. Aho, L. Sumanen, M. Waltari, and K. Halonen, "A 14b 200MHz IF-sampling A/D converter with 79.9dB SFDR," IEEE Norchip Conference Proceeding, pp. 171-174, November 2004.

[3] P. Bogner, F. Kuttner, C. Kropf, T. Hartig, M. Burian, and H. Eul, "A $14 \mathrm{~b} 100 \mathrm{MS} / \mathrm{s}$ digitally self-calibrated pipelined ADC in $0.13 \mu \mathrm{m}$ CMOS," IEEE International Solid-State Circuits Conference Digest of Technical Papers, pp. 224-225, 650, February 2006.

[4] S. M. Jamal, D. Fu, N. C.-J. Chang, P. J. Hurst, and S. H. Lewis, "A 10-bit $120 \mathrm{MSample} / \mathrm{s}$ time-interleaved analog-to-digital converter with digital background calibration," IEEE Journal of Solid-State Circuits, vol. 37, pp. 1618-1627, December 2002.

[5] H.-C. Liu, Z.-M. Lee, and J.-T. Wu, "A 15-b 40-MS/s CMOS pipelined analog-to-digital converter with digital background calibration," IEEE Joumal of Solid-State Circuits, vol. 40, pp. 1047-1056, May 2005.

[6] C.-C. Hsu and J.-T. Wu, "A CMOS 33-mW 100-MHz 80-dB SFDR sample-and-hold amplifier," IEICE Transactions on Electronics, vol. E86C, pp. 2122-2128, October 2003. 